\title{
THE ORDER OF THE IMAGE OF THE $J$-HOMOMORPHISM
}

\author{
BY MARK MAHOWALD
}

Communicated by Raoul Bott, June 4, 1970

ABstract. This note announces a proof of the order of the image of the $J$-homomorphism and gives several other results in homotopy theory which are consequences of the proof.

The set $\Omega^{n} S^{n}$ can be identified with the set of all base point preserving maps of $S^{n}$ into itself. $S O(n)$, acting on $S^{n}$ as $R^{n}$ with a point at infinity, is also a set of base point preserving maps of $S^{n}$ onto itself. This defines $S O(n) \subset \Omega^{n} S^{n}$. The induced map in homotopy is called the $J$-homomorphism. If we allow $n$ to go to infinity we have the stable $J$-homomorphism. By Bott's results [3] $\pi_{j}(S O)=Z$, $j \equiv-1 \bmod 4$, and $=Z_{2} j \equiv 0,1 \bmod 8, j>0$, and zero otherwise. Adams [1] showed that the $Z_{2}$ summand maps monomorphically and Milnor and Kervaire [6] showed that the $Z$ group in dimension 4j-1 maps nontrivially and its image generates a subgroup of at least a certain order $\lambda_{j}^{\prime}$. Adams [1] showed that the order was either $\lambda_{j}^{\prime}$ or $2 \lambda_{j}^{\prime}$ and if $j \equiv 1$ (2) it was $\lambda_{j}^{\prime}$. Thus only the two primary part is in question and there only for $j \equiv 0(2)$. Let $\lambda_{j}$ be the two primary part of $\lambda_{j}^{\prime}$. If $4 j \equiv 2^{\rho(j)} \bmod 2^{\rho(j)+1}$ (which defines $\rho(j)$ ) then $\lambda_{j}=2^{\rho(j)+1}$. We prove:

THEOREM 1. The 2-primary order of the image of $J$ in stem $4 j-1$ is $\lambda_{j}$.

The proof has several corollary results which have some interest. The first result is rather technical but still has some interest. The naming of elements in $H^{* *}(A)$ is that given in [5].

Theorem 2. The elements $P^{i} c_{0}, P^{i} h_{1} c_{0}, i \geqq 1, P^{i} h_{2}, i \geqq 1$, in $H^{* *}(A)$ represent the image of $J$ in dimension $j \equiv 0,1,3 \bmod 8$. In dimension $8 j-1$ the "tower" which ends at the "Adams edge" represents the image of $J$ in that dimension.

These elements were known to have the desired $e$-invariant property [1] and were believed to be in $J$. Their Whitehead product behavior has been investigated ([2] and [4], for example).

Let $M=Z_{2}+Z_{2}$ (be the module over $A$ with one generator; $\mu$ in

AMS 1970 subject classifications. Primary 55E10, 55E50, 55H15.

Key words and phrases. Stable homotopy groups of spheres, $J$-homomorphism, cohomology of the Steenrod algebra. 
dimension zero and $S q^{1} \neq 0$. Let $P\left(x_{1}, \cdots\right)$ be a polynomial algebra on generators $x_{i}$ with bidegree $\left(2,2^{i+2}+1\right)$. Consider the differential $d\left(x_{i}\right) \rightarrow x_{i-1}^{2} x_{1}$ in $P$. Let $H(d)$ be the homology under $d$ and $B(d)$ $=\operatorname{im} d$.

For $\alpha \in P$ let the bidegree of $\alpha$ be $\left(\alpha_{s}^{\prime}, \alpha_{t}^{\prime}\right)$. We will be only interested in the values of $\alpha_{s}^{\prime}$ modulo 4 and $\alpha_{t}^{\prime}$ modulo 12 so take $\left(\alpha_{s}, \alpha_{t}\right)$ so that $\alpha_{s} \equiv \alpha_{s}^{\prime}(\bmod 4), \alpha_{t} \equiv \alpha_{t}^{\prime}(\bmod 4)$ but $5 \alpha_{s}<\alpha_{t}-\alpha_{s}$.

TheOREM 3. If $5 s \geqq t-s+\epsilon$ where $\epsilon$ depends on the congruence class of $s \bmod 4$ and $\epsilon \leqq 6$, then

$$
\begin{aligned}
\operatorname{Ext}_{A}^{s, t}\left(M, Z_{2}\right)= & \sum_{\alpha \in H(d)} \operatorname{Ext}_{A_{0}}^{s-\alpha_{s}, t-\alpha_{t} t}\left(M \otimes A / / A_{1}, Z_{2}\right) \\
& \oplus \sum_{\alpha \in B(d)} \operatorname{Ext}_{A}^{s-\alpha_{s}, t-\alpha t}\left(M \otimes A / A\left(S q^{3}, S q^{1}\right), Z_{2}\right) .
\end{aligned}
$$

Corollary 4. If $Q$ is an $A$ module which is free over $A_{1}$, the subalgebra generated by $S q^{1}$ and $S q^{2}$, then $\operatorname{Ext}_{A}^{s, t}\left(Q, Z_{2}\right)=0$ for $5 s \geqq t-s+\epsilon$.

THEOREM 5. Let $X$ be a space in the stable category so that $\Sigma X=R P^{2}$. If $E_{r}(X)$ is the Adams spectral sequence converging to $\pi_{*}^{S}(X)$, then then $E_{5}^{s, t}=E_{\infty}^{s, t}(X)=0$ for $5 s \geqq t-s+\epsilon$ unless

$$
\begin{aligned}
s & =4 k, \quad t-s=8 k, \quad 8 k+1, \quad 8 k+2, \\
& =4 k+1, \quad t-s=8 k+1, \quad 8 k+2, \quad 8 k+3, \\
& =4 k+2, \quad t-s=8 k+2, \quad 8 k+3, \quad 8 k+7, \\
& =4 k+3, \quad t-s=8 k+4, \quad 8 k+8, \quad 8 k+9,
\end{aligned}
$$

in which cases the groups are $Z_{2}$.

These elements represent the generators of the image of $J$ and $\mu_{j}$ [1] on the bottom cell and the elements of order two in the im $J$ and $\mu_{j}$ coextended on the top cell.

THeORem 6. There is a space $\operatorname{Im} J$ and a map $f: S^{0} \rightarrow \operatorname{Im} J$ so that $f_{*}$ maps the image of $J$ and the $\mu$ 's monomorphically onto the homotopy of $\operatorname{Im} J$.

In [1] a map $f: \Sigma^{8} X \rightarrow X$ which represents an extension of a coextension of $8 \sigma$ is studied. There it is proved that all iterations of $f$ are essential.

TheOREM 7. If $\alpha: S^{k} \rightarrow X$ is a stable map then

$$
S^{k+8 j} \underset{\Sigma^{8 j} \alpha}{\longrightarrow} \Sigma^{8 j} X \underset{f^{j}}{\rightarrow} X
$$


is inessential for some $j$ unless $\alpha$ is in one of the classes given by Theorem 5.

Some comments on the proof. Let the spectrum bo be the connected $B O$ spectrum. Then we construct a Novikov resolution of $S$ as follows

$$
\begin{aligned}
& \dot{:} \\
& \dot{S_{\sigma}} \rightarrow S_{\sigma} \wedge b o \\
& \dot{S_{1}} \rightarrow S_{1} \wedge b o \\
& S \rightarrow S \wedge b o .
\end{aligned}
$$

We apply the $E_{2}$ of the Adams spectral sequence to this tower and get a spectral sequence which converges to $H^{* *}(A)$ except for $s=t$. If we consider the resolution $X \wedge S_{\sigma}$, where $X$ is defined in Theorem 5 , we can make an explicit calculation. Let

$$
E_{1}^{s, t \sigma}=\operatorname{Ext}_{A}^{s-\sigma, l-\sigma}\left(\tilde{H}^{*}\left(X \wedge S_{\sigma} \wedge b o\right), Z_{2}\right) .
$$

Proposition 8. $E_{2}^{s, t, \sigma}=\sum_{\alpha \in P^{\sigma}} \operatorname{Ext}_{A}^{s-\alpha_{t, t}-\alpha_{t}}\left(M \otimes A / / A_{1}, Z_{2}\right)$ for $s>\sigma$ where $P^{\sigma}$ is the set of $\sigma$-degree polynomials in the polynomial algebra introduced above.

Proposition 9. $E_{3}^{s, t, \sigma}=E_{\infty}^{s, t, \sigma}$ for $s>\sigma$ and thus is given by Theorem 3.

Note that Proposition 9 alone gives an edge of $3 \sigma>t-2$. The sharpened version of Theorem 3 follows from Proposition 9 and a closer analysis of the nature of $\operatorname{Ext}_{A}^{s, t}\left(M, Z_{2}\right)$.

The most direct route from Proposition 8 to the main theorem requires a geometric realization of the $E_{2}$ term of the above spectral sequence for $S$. Using this resolution and the homotopy functor we get a spectral sequence whose $E_{2}^{\sigma, t}$ term has an edge of $5 \sigma \geqq t-\sigma+\epsilon$. The image of $J$ has filtration 1. From this information the order of im $J$ should follow directly but no direct route has been found. Hence to complete the argument, consider the space $Y$ which is the fiber of the map $S \rightarrow K(Z, 0)$, and consider the resolution of $Y$ given by $\cdots \rightarrow Y \wedge S_{\sigma} \rightarrow Y \wedge S_{\sigma-1} \rightarrow \cdots$. Calculation of the sort given in the proof of III 7.3 of [4] and applied to elements of filtration zero and one give a proof of Theorems 1 and 2 .

\section{REFERENCES}

1. J. F. Adams, On the groups $J(X)$. IV, Topology 5 (1966), 21-71. MR 33 \#6628.

2. D. W. Anderson, The e-invariant and the Hopf invariant, Topology 9 (1970), 49-54. 
3. R. Bott, The stable homotopy of the classical groups, Ann. of Math. (2) 70 (1959), 313-337. MR 22 \#987.

4. M. E. Mahowald, The metastable homotopy of $S^{n}$, Mem. Amer. Math. Soc. No. 72 (1967). MR 38 \#5216.

5. M. E. Mahowald and M. C. Tangora, Some differentials in the Adams spectral sequence, Topology 6 (1967), 349-369. MR 35 \#4924.

6. J. W. Milnor and M. A. Kervaire, Bernoulli numbers, homotopy groups, and a theorem of Rohlin, Proc. Internat. Congress Math. (Edinburg, 1958) Cambridge Univ. Press, New York, 1960, pp. 454-458. MR 22 \#12531.

Northwestern University, Evanston, Illinois 60201 